\title{
Los videojuegos como herramienta para el fomento a la lectura de textos literarios
}

\author{
(Video Games as a Tool for the Promotion of Reading Literature)
}

\author{
Adriana Munguía López, Universidad de Sonora, Sonora, México \\ María Edith Araoz Robles $\oplus$, Universidad de Sonora, Sonora, México
}

\section{Volumen 1, Número 1}

Edición especial. Febrero de 2016

p. $162-178$

Este número se publicó el 5 de febrero de 2016

Artículo recibido: 9 de marzo de 2015

Artículo aprobado: 5 de noviembre de 2015

ISSN: 2448-5942, doi: https://doi.org/10.36799/el.v1i1.27

Munguía López, A., \& Araoz Robles, M. E. (2016). Los videojuegos como herramienta para el fomento a la

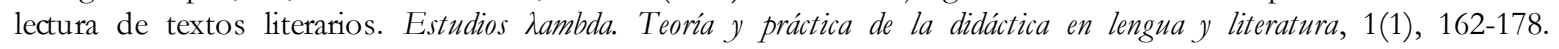
$\underline{\text { https://doi.org/10.36799/el.v1i1.27 }}$

Derechos de autor: El autor o autores conservan en todo momento sus derechos morales y patrimoniales sobre la obra; la obra no se puede alterar, transformar o ampliar; siempre debe reconocerse la autoría del documento

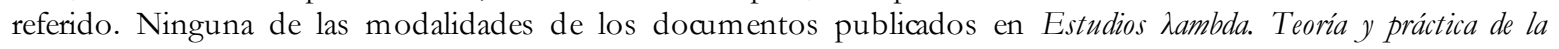
didáctica en lenguay literatura tienen fines comerciales de naturaleza alguna.

Los contenidos de este artículo están bajo una licencia de Creative Commons Atribución no comercial- Sin

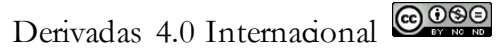




\title{
Los videojuegos como herramienta para el fomento a la lectura de textos literarios
}

\author{
(Video Games as a Tool for the Promotion of Reading Literature)
}

\author{
ADRIANA MUNGUÍA LÓPEZ ${ }^{1}$ \\ MARÍA EDITH ARAOZ ROBLES ${ }^{2}$
}

\section{RESUMEN}

Tomando en cuenta la situación de lectura en el país, en la que, según estudios y encuestas oficiales (como la realizada por el Consejo Nacional para la Cultura y las Artes en 2006) no sólo es mínimo el hábito lector, sino que quienes se dedaran lectores no necesariam ente lo son de textos literarios, en esta ponencia presentamos una propuesta acerca de la utilización de videojuegos comerciales como una nueva herramienta para acercar a los jóvenes a la literatura. Este trabajo es parte de una investigación de tesis que se está realizando en la que confluyen dos fenómenos en apariencia diferentes y de mundos separados: la literatura y los videojuegos, considerados estosúltimos como un tipo de narración audiovisual. Nuestro objetivo aquí es dar a conocer aspectos importantes del taller literario que se llevó a cabo con jóvenes universitarios, con la finalidad de despertar en ellos el interés hacia la literatura utilizando su afición a los videojuegos comerciales. Aun cuando los resultados obtenidos en dicho taller están en proceso de análisis, un primer acercamiento nos permite observar que los videojuegos pueden ser una herramienta eficaz para fom entar el gusto por la literatura.

PALABRAS CLAVE: Fom ento a la lectura, videojuegos, literatura.

\begin{abstract}
In the context of present reading situation in Mexico, studies published by official institutions (Consejo Nacional para la Cultura y las Artes in 2006), report that the reading habit in the country, not only is minimal, but even that those who call themselves readers not necessarily read literature. In this paper the authors present a proposal on the use of commercial video games as a tool to approach young gamers to literature. This work is a part of a thesis investigation under way, where two phenomena, apparently different to each other and from different worlds, converge: literature and video games, this last, considered as visual narrative. Our goal is to present the important aspects of the literary workshop that took place at University of Sonora with university students, in order to rouse their interest in the literary world using their attachment for video gam es. Although the results of this work are still in the process of analysis, a first approach allows us to observe that video games can be an effective way to promote literature and encourage reading habits.
\end{abstract}

KEYWORDS: Reading promotion, video games, literature.

\footnotetext{
${ }^{1}$ Lic, en Literaturas Hispánicas, maestrante en el posgrado en Humanidades del Departamento de Letras y Lingüística de la Universidad de Sonora. adriana.mun@gmail.com

2 Maestra de tiempo completo del Departamento de Letras y Lingüística de la Universidad de Sonora. Candidata a doctor en ciencias sociales. Líneas de investigación: comprensión lectora y producción textual; y, construcción de sentid: discurso, identidades e imaginarios. edith.araoz@unison.mx
}

Artículo recibido: 9 de marzo de 2015

Aprobado: 5 de noviembre de 2015

Munguiay Araoz. doi: https://doi.org/10.36799/el.v1i1.27 Volumen 1, Número 1, Año 2016, ISSN: $2448-5942$ 


\section{INTRODUCCIÓN}

Hoy en día, la irrupción de los medios de comunicación audiovisuales ha creado un nuevo tipo de lector que es atraído por las nuevas tecnologías y por el uso cada vez mayor de las redes sociales y los videojuegos. Vemos así a niños y jóvenes que crecen sin el hábito de la lectura, pues prefieren sentarse frente a una pantalla de televisión o frente al monitor de una computadora a participar en redes sociales o a jugar su videojuego favorito. El placer de leer una obra literaria se ha dejado de lado por considerarla una actividad obligatoria relacionada sólo con tareas escolares.

Este panorama resulta desalentador si reconocemos que la lectura literaria es un elemento de suma importancia para el desarrollo del individuo. Además del goce estético, a través de la literatura podemos percibir otras formas de interpretar el mundo, distinguir puntos de vista diferentes sobre un mismo tema o encontrar otras voces que nos van enriqueciendo tanto intelectual como moralmente. De igual manera, como menciona Cassany $(2007,194)$, aspectos tan importantes como el éxito o el fracaso escolar, la preparación técnica para acceder al mundo del trabajo o el grado de autonomía y desenvoltura personal están estrechamente relacionados con las capacidades que el individuo desarrolla por medio de la lectura.

Con base en la problemática aquí planteada, en este trabajo se presenta una nueva estrategia para el fomento a la lectura: los videojuegos. Nuestro interés es demostrar que éstos, en lugar de ser sólo un entretenimiento o distractor negativo para los estudiantes, pueden ser un medio para fomentar el gusto por la lectura literaria. Coincidimos con Lluch en que "La idea no es condenar o desprestigiar sino descubrir capacidades ignoradas que nos ayuden a crear con ellas un puente" $(2012,156)$.

Partimos del supuesto de que los videojuegos pueden ser utilizados como herramienta educativa no sólo para desarrollar habilidades lógico-matemática y motriz, sino también como estrategia para la didáctica de la literatura. Los videojuegos pueden utilizarse como instrumentos de aprendizaje y formación de lectores, toda vez que:

- tienen una fuerte similitud e influencia de la literatura, especialmente por la narración,

Munguiay Araoz doi: https://doi.org/10.36799/el.v1i1.27 Volumen 1, Número 1, Año 2016, ISSN: $2448-5942$ 
- son narraciones interactivas y contienen los elementos básicos de un texto literario tales como la historia, su narrativa, tipos de personajes, temáticas...,

- son una especie de libros electrónicos interactivos con apoyo multimedia,

- llaman la atención y tienen una gran aceptación por parte de niños, jóvenes y adultos,

- son conocidos y practicados por un buen número de estudiantes que han crecido conviviendo con ellos día a día.

Esta propuesta forma parte de una investigación de tesis que gira en torno a la cuestión del fomento a la lectura. Se presenta aquí el estudio de una nueva herramienta para atraer lectores o para lograr la aceptación de la lectura de textos literarios en jóvenes que muestran apatía hacia esta actividad o que no han tenido contacto significativo con la literatura. Para ello se reunieron dos fenómenos aparentemente diferentes y de mundos separados: la literatura y los videojuegos.

Como parte de la propuesta, se desarrolló un taller literario en el que se utilizaron videojuegos comerciales, videojuegos con los cuales los jóvenes comúnmente interactúan y son de su interés. Para su elección, se consideraron aspectos relativos a su temática, al género, a los personajes, a la narración y al tiempo-espacio en el que se desarrollan. De igual manera, se buscó que tuvieran alguna relación con obras literarias o que contaran con elementos similares. El taller se enfocó a trabajar con estudiantes universitarios aficionados a los videojuegos y que tuvieran experiencia en jugarlos.

Para explicar el proceso seguido en el desarrollo del taller, estructuramos este trabajo en tres apartados. En primer término, y a manera de justificación del taller, se exponen algunos antecedentes y datos relevantes que nos brindan un panorama general de la situación de la lectura en México así como de los tipos de lectores y de lecturas. Posteriormente, se describe el proceso seguido en la realización del taller, sus objetivos y metodología. Por último, se mencionan los resultados obtenidos. Iniciamos enseguida con la panorámica sobre la situación lectora.

Munguiay Araoz doi: https://doi.org/10.36799/el.v1i1.27 Volumen 1, Número 1, Año 2016, ISSN: $2448-5942$ 


\section{ANTECEDENTES GENERALES SOBRE LA SITUACIÓN DE LA LECTURA EN MÉXICO}

El interés en realizar esta investigación contextualizada dentro del fomento a la lectura ${ }^{3}$ surge al reflexionar sobre la situación de lectura en el país. Al revisar las cifras presentada s por encuestas y estudios, es posible advertir que los mexicanos no tenemos arraigado el hábito de la lectura. Si bien los estudios y encuestas presentan porcentajes de alguna manera positivos, los datos encontrados nos dicen que la lectura que se realiza no necesariamente corresponde a la lectura de textos literarios. Revisemos algunos de estos datos.

Con motivo del día internacional del libro, Jenaro Villamil publicó en la revista Proceso, en abril de 2013, datos de la UNESCO sobre el índice de lectura en México: "Entre 108 países, México es penúltimo lugar en lectura”. Asimismo, la Fundación Mexicana para el Fome nto a la Lectura publicó en 2012 un estudio titulado "De la penumbra a la oscuridad", en él presenta cifras proporcionadas por la Encuesta Nacional de Lectura (ENL), encuesta realizada a su vez por el Consejo Nacional para la Cultura y las Artes. De acuerdo con este estudio, se reporta una disminución de lectores: en 2006 el 56\% de la población mexicana se declaraba lectora, mientras que en 2012 sólo fue el 46\%. Además de esto, se exhibe también que la lectura se sigue considerando como una actividad escolar. El hábito de la lectura se registra principalmente en jóvenes de 12 a 17 años y se considera que los mexicanos dejan la lectura al momento que concluir sus estudios. En 2012, de la población que se declaró ser lectora, el $42 \%$ dice leer libros escolares, el $22 \%$ lee libros sobre historia y el 18\% lee novelas.

En cuanto al tiempo dedicado a la lectura, el estudio menciona que el 29\% lee dos horas o menos a la semana, el $21 \%$ de tres a cinco horas y el $16 \%$ lee seis o más horas semanales. Respecto a la cantidad de libros leídos al año, en promedio es de 2.9 libros; la principal razón por la cual se lee es estar informado $(24.6 \%)$ y por motivos escolares $(20.5 \%)$. Solo el 9.2\% declara leer por gusto. Además, conforme aumenta la edad, la actividad lectora

\footnotetext{
3 Al hablar de fomento a la lectura hacemos referencia a las distintas actividades y estrategias que buscan, como el mismo término lo dice, fomentar la lectura, impulsarla, promoverla. Significa también presentar la lectura e introducir en ella a los nuevos lectores; asimismo implica promover y reforzar la actividad entre quienes ya leen de manera habitual.
}

Munguiay Araoz, doi: https://doi.org/10.36799/el.v1i1.27 Volumen 1, Número 1, Año 2016, ISSN: $2448-5942$ 
disminuye. A la pregunta “¿A qué edad cree usted que leía más?” un 28.5\% contestó que entre los 12 y 14 años; el 19.3\%, entre los 15 y 17 años; el 10\% entre los 18 y 22 años, y por último el $3.7 \%$ entre los 23 y 25 años. En vista de tales cifras, es notable que la actividad lectora se realiza principalmente durante los años escolares, además de que el material de lectura no es precisamente de textos literarios, sino libros escolares o relacionados con su campo de estudio o trabajo.

$\mathrm{Al}$ revisar el escenario de lectura en el país es factible advertir la situación en la que se encuentra la lectura actualmente, además de distinguir diferentes tipos de lectura y de lectores. Adela Castronovo en su libro Nuevas propuestas en promoción de la lectura (2007) menciona la existencia de los lectores, los poco lectores y los no lectores. Quienes se distinguen como lectores son los que ya han sido cautivados por la lectura y la idea de descubrir sus mundos posibles, son quienes realizan la lectura por iniciativa propia y por gusto. Los poco lectores son aquellos que leen pero no han desarrollado un hábito lector. "Leen de manera precaria y discontinua" (11). Por último, los no lectores son quienes no han tenido relación alguna con la lectura ni muestran interés en esta actividad.

Por su parte, autores como Rosa Ana Martín Vegas en su libro Manual de didáctica de la lengua y la literatura (2010) y Pedro Cerrillo en su artículo "Los nuevos lectores" (2007), conciben la existencia de dos tipos de lectores. De acuerdo con Martín Vegas, uno es el lector tradicional "que lee todo tipo de libros, dependiendo de sus gustos y entiende la lectura como una fuente de conocimiento y de placer" (307). Mientras que existe un nuevo lector, que

lee prácticamente sólo documentos informativos para su trabajo o de uso en su profesión, textos informativos en la red de carácter divulgativo sobre cuestiones prácticas, publicidad, paneles... y lo estrictamente necesario para su vida, para saciar su curiosidad o llenar su tiempo. El perfil del llamado "nuevo lector" se caracteriza porque no lee en profundidad ningún texto: simplemente recorre sus párrafos por encima leyendo y comprendiendo las palabras resaltadas tipográficamente o centrándose en las imágenes que ilustran los temas. Este "nuevo lector" maneja p ocos libros, pasa horas delante del ordenador enchufado a la Red. (2010, 307).

Munguiay Araoz doi: https://doi.org/10.36799/el.v1i1.27 Volumen 1, Número 1, Año 2016, ISSN: 2448-5942 
Para Cerrillo, el lector tradicional es un lector competente, habituado a todo tipo de lectura, desde lo literario hasta de los nuevos modelos impulsados por las nuevas tecnologías $(2007,2)$. El nuevo lector es aquel que levanta su bandera de buen lector porque lee todo el tiempo, lee en la escuela, lee en el trabajo, lee mientras navega por internet, etcétera. Cerrillo lo describe como un consumidor que se encuentra enganchado a las nuevas tecnologías, sólo lee información, datos e incluso publicidad. Este tipo de lector no contempla los textos narrativos como su principal fuente de lectura. Su experiencia lectora se remonta a sus experiencias con el cine, televisión y textos escolares (2).

Así como existen tipos de lectores, también es posible hablar de tipos de lectura. Cerrillo hace una división referente a la lectura que realizan los lectores. Menciona la existencia de dos tipos de lectura: la lectura obligatoria y la voluntaria. La primera es una lectura totalmente interesada y dirigida donde el lector no tiene libertad de elección, es una obliga ción escolar que cumplir y, por lo general, sólo se trata de fragmentos aislados de alguna obra literaria. La segunda, la lectura voluntaria, se basa en la disposición personal del lector y es totalmente libre e inclinada al ocio. ${ }^{4} \mathrm{Al}$ ser una lectura libre, el lector se ve envuelto en mundos imaginarios y se le facilita la interpretación del mundo real (6).

Es común la idea de otorgar a la escuela el deber de desarrollar el hábito lector en los alumnos, en ella se desarrollan la mayoría de las actividades de animación a la lectura: en sus bibliotecas, entre clases, programas de lectura, bibliotecas móviles, ferias de libro, entre otras. Aunque estos esfuerzos sí logran mostrar algunos resultados positivos, no son suficientes, pues como ya vimos, la lectura se sigue registrando sólo durante la etapa escolar y no de manera voluntaria, no se adquiere el hábito de leer fuera de la escuela ni la voluntad por leer. Niños y jóvenes prefieren sentarse frente al monitor de la computadora a participar en redes sociales o frente al televisor. Cerrillo denomina este fenómeno como un neoanalfabetismo, "desarrollado y protagonizado por esos nuevos lectores, fascinados por los nuevos soportes de lectura, que no son lectores literarios ni tampoco, en muchos casos, lectores competentes." (9). Este neoanalfabetismo, nos dice, sólo puede ser superado mediante la lectura de textos literarios.

\footnotetext{
${ }^{4} \mathrm{Al}$ referirse a la lectura como una actividad inclinada al ocio o improductiva, Cerrillo la entiende como una actividad fuera de lo laboral-escolar. Una actividad que a diferencia de la lectura escolar, no busca obtener una calificación o aprobar un curso, sino una actividad libre, sin una función determinada más que el mismo goce estético que el lector experimenta al enfrentarse voluntariamente con una obra literaria.
}

Munguiay Araoz. doi: https://doi.org/10.36799/el.v1i1.27 Volumen 1, Número 1, Año 2016, ISSN: $2448-5942$ 
Ahora bien, ¿Por qué la importancia y la insistencia por fomentar la lectura de textos literarios? Como se mencionó, consideramos que la lectura de obras literarias es un elemento importante para el desarrollo del individuo pues al leerlas percibe formas diferentes de interpretar el mundo, distintos puntos de vista sobre un mismo tema; encuentra otras voces que lo van enriqueciendo tanto intelectual como moralmente. Al mismo tiempo se va educando en las formas estéticas, formas que permiten apreciar la expresión de sentimientos y pensamientos. Estas características son significativas para el desarrollo y formación del individuo.

Coincidimos con Cassany en que "La lectura es un instrumento potentísimo de aprendizaje: leyendo libros, periódicos o papeles podemos aprender cualquiera de las disciplinas del ser humano.”(2007, 193). Para Cassany además de la adquisición del código escrito, el lector desarrolla capacidades cognitivas superiores como la reflexión y el espíritu crítico. La lectura de textos literarios trae consigo múltiples beneficios a quien se introduce en ella, como una formación integral y humanizada, capacidad de reflexión y sensibilización, además del desarrollo de un criterio propio y conocimientos de cultura en general.

De acuerdo con García Galeano, existen dos aspectos principales que señalan la "utilidad" de la literatura: "La literatura en tanto que es encarnación estética de los "valores eternos" del hombre, enriquecería espiritualmente al educando". "La literatura, el arte en general, puede operar en la sociedad para transformarla". (2001, 339). Asimismo, existen beneficios más "útiles" a ojos prácticos. Como la adquisición y desarrollo de competencias y habilidades en expresión oral, escrita y literaria. La capacidad de desarrollar plenamente la lengua materna e incluso una extranjera. "Formar hablantes competentes y lectores eficaces, buenos redactores, individuos plenamente desarrollados y capaces de relacionarse con los demás" (11).

Así pues, consideramos importante fomentar la lectura literaria para crear individuos críticos que actúen por convicción propia, que sepan emitirjuicios propios y reflexionar sobre sus acciones y las de los demás. Consideramos también que es necesaria la búsqueda de nuevas estrategias para acercar a los individuos a la lite ratura. En este proyecto se hace la propuesta de una nueva estrategia para acercar a los jóvenes a la lectura de textos literarios. Dicha propuesta se basa principalmente en el uso de videojuegos, en el gusto que los jóvenes tienen hacia tal Munguiay Araoz doi: https://doi.org/10.36799/el.v1i1.27 Volumen 1, Número 1, Año 2016, ISSN: 2448-5942 
actividad y en el conocimiento previo con que ellos cuentan. Como parte de esta propuesta, se describe enseguida la realización del taller.

\section{TALLER DE LITERATURA Y VIDEOJUEGOS}

El taller de literatura y videojuegos consistió en construir un puente entre el mundo de los videojuegos (ya conocido por los jóvenes) y el mundo literario (con el que no están familiarizados); en lograr el acercamiento de los estudiantes con la literatura, para que la lectura de textos literarios no sea considerada una obligación escolar, sino una actividad tan placentera como jugar videojuegos. ${ }^{5}$

Objetivos del taller. El taller de videojuegos y literatura busca introducir a los estudiantes al mundo de la literatura, romper la barrera que comúnmente existe entre los jóvenes y los textos literarios, fomentar el gusto por la lectura o, al menos, lograr un cambio de actitud positivo hacia la literatura.

\section{METODOLOGÍA}

Para la realización del taller, dado que su objetivo es fomentar la lectura de textos literarios por medio de videojuegos comerciales, se tomaron como sujetos de estudio a un grupo de estudiantes de la Universidad de Sonora que tuvieran poca o ninguna experiencia literaria pero sí experiencia e interés en jugar videojuegos.

La decisión de incorporar a estudiantes universitarios en este taller obedeció a varias razones. Una de ellas fue aprovechar el calendario escolar (en vacaciones es difícil que se incorporen), otra, fue la oportunidad de utilizar el sistema CULTUREST de la Universidad de Sonora (ello aseguraba la asistencia constante de los inscritos al taller). Otra razón, no menos importante, es que se logró incorporar el taller a los talleres literarios que organiza la Biblioteca

\footnotetext{
${ }^{5}$ El término videojuegos es entendido aquí como un dispositivo electrónico mediante el cual uno o varios jugadores toman parte primordial en una narración interactiva y en donde el jugador deberá seguir las reglas establecidas por el mismo juego, resolver problemas e incluso interactuar con otros personajes y o jugadores. En este caso no importa si se trata de un juego ejecutado en una computadora o videoconsola casera o portátil, (lo cual forma parte de los debates acerca de la especificación del término).

Munguiay Araoz, doi: https://doi.org/10.36799/el.v1i1.27 Volumen 1, Número 1, Año 2016, ISSN: $2448-5942$
} 
Fernando Pesqueira. ${ }^{6}$ Además, un grupo de estudiantes universitarios cubre las condiciones necesarias, ya que por la edad, 18 a 25 años aproximadamente, han tenido más oportunidad de convivir y desenvolverse en videojuegos. Por último, cabe destacar que este taller se inclinó por trabajar con alumnos universitarios porque comúnmente las acciones y la preocupación por crear nuevos lectores se enfoca principalmente en niños pequeños, en adolescentes y muy poco en los jóvenes-adultos. ${ }^{7}$

El taller de literatura y videojuegos se anunció y se convocó desde el 2 de mayo de 2014, por medio de carteles colocados en distintos puntos de la Universidad de Sonora. Durante una semana se recibió respuesta de alumnos interesados, un total de 13 jóvenes, seis varones y siete mujeres. De éstos empezaron el taller nueve, pero el número cambió con los días al interesarse amigos de los estudiantes asistentes y al ausentarse uno. Finalmente quedaros once jóvenes, 7 varones y 4 mujeres. ${ }^{8}$ Éstos se dividieron en dos grupos por cuestiones de horario. Se trabajó con el primer grupo durante las mañanas de 11:00 am a 13:00 pm y con el segundo grupo durante la tarde de 15:00 pm a 17:00 pm.

El taller se llevó a cabo en la Biblioteca Fernando Pesqueira durante los meses de junio y julio de 2014, iniciando el día martes 3 de junio y finalizando el día martes 8 de julio. Se realizaron 11 sesiones de dos horas cada una, dos veces a la semana. La realización del taller se dio en dos etapas con el fin de introducir poco a poco al estudiante al mundo literario.

En la primera etapa, denominada narrativa y videojuegos, se trató de acercar a los estudiante a la narración a través de los videojuegos comerciales, hacerlos conscientes de la presencia de la narración en medios audiovisuales, en este caso, en los videojuegos que comúnmente juegan, para que reconocieran en ellos elementos narrativos tales como tiempo,

\footnotetext{
${ }^{6}$ En estos talleres participan estudiantes de todas las licenciaturas de la Universidad de Sonora. A cambio de asistir a ellos, se les certifican puntos necesarios para acreditar el programa CULTUREST. Es una forma de convocar e interesar a individuos no lectores a un taller literario.

${ }^{7}$ Es importante prestar atención a este grupo, toda vez que, como se observó en los estudios presentados, la actividad lectora es registrada principalmente en el ámbito escolar y va disminuyendo conforme se avanza en edad o bien se abandona al concluir los estudios. Además, la mayoría de los programas educativos universitarios no contemplan en sus planes de estudio asignaturas de lengua o literatura, la actividad lectora del alumno se concentra en textos científicos y académicos y deja de lado los textos literarios.

${ }^{8}$ De estos 11 jóvenes se dieron casos particulares: un joven interesado en el taller, ajeno a la Universidad de Sonora fue introducido a los talleres literarios por la maestra Sofía Rayas, quien al advertir su gran interés y experiencia en videojuegos lo recomendó para el proyecto. Este mismo joven intentó integrar a un amigo, quién asistió sólo a tres sesiones. Otro caso fue el de un joven egresado de la licenciatura en diseño gráfico que se integró al taller a los días de haber iniciado, por recomendación de una de las estudiantes asistentes. A diferencia del resto de los talleristas, él tenía experiencia tanto en los videojuegos como en la lectura de textos literarios.
}

Munguiay Araoz. doi: https://doi.org/10.36799/el.v1i1.27 Volumen 1, Número 1, Año 2016, ISSN: $2448-5942$ 
espacio, personajes, narrador y la estructura. Se trabajó también el conocimiento previo que el estudiante tiene sobre éstos y se realizaron además actividades de escritura creativa, discusiones y debates, comparaciones con otro tipo de narrativa (cine, televisión, literatura y otros videojuegos).

En la segunda etapa, designada como literatura y videojuegos los estudiantes entraron en contacto directo con la literatura y se familiarizaron con el mundo narrativo que se encuentra en los videojuegos. Después de haber reflexionado sobre la narrativa en los videojuegos, se le proporcionaron textos literarios cortos con elementos y temáticas relacionados con los videojuegos para que empezara a establecer conexiones y comparaciones entre estos dos mundos, e incluso con otras formas de narrativa.

La primera etapa también se aprovechó para conocer a los estudiantes, indagar sobre sus gustos y sobre los videojuegos con los que se encuentren más familiarizados. De acuerdo con Castronovo (2007), este acercamiento es necesario ya que, en el fomento a la lectura, el factor de mayor importancia es conocer al posible lector para construir una idea aproximada de los textos narrativos que se le pueden ofrecer: "En primer lugar, hay que indagar las características del posible lector, de aquel que anda buscando el rumbo en la lectura y para quien el próximo libro a leer puede significar seguir construyéndose como lector o abandonar el intento por aburrimiento, por dificultad o por desinterés. (57).

Esta autora propone cuatro puntos esenciales a observar en el posible lector: la edad, frecuentación lectora, intereses y lecturas previas. Castronovo menciona que la edad debe ser un punto de partida para determinar los materiales literarios a ofrecer, pero no debe ser un punto determinante. La frecuentación lectora - es decir, el tiempo y los momentos dedicados a la lectura-es importante ya que indica qué tanta disposición a leer tiene la persona. También es necesario prestar especial atención a los intereses del posible lector, ya que no siempre resulta positivo recomendar una lectura sin tomar en cuenta los gustos del individuo que apenas se inicia en esta actividad. Conviene asimismo tener conocimiento de las lecturas previas del futuro lector, ya que pueden servir como guía para quién recomienda la lectura (57-59).

Queda clara así la necesidad de conocer a los alumnos con los que se trabajará durante el taller y analizar sus características: actividad lectora previa, y sobre todo los intereses, ya que

Munguiay Araoz doi: https://doi.org/10.36799/el.v1i1.27 Volumen 1, Número 1, Año 2016, ISSN: $2448-5942$ 
"se trata en todo caso, de tender un puente entre ambos, de crear un vínculo, de concretar un nexo. Pero también se trata de ayudar a construirlos" (59).

Estos elementos mencionados anteriormente: edad, frecuentación lectora, intereses y lecturas previas, fueron compilados y analizados mediante la aplicación de un cuestionario a los integrantes del taller de literatura y videojuegos. Dentro del cuestionario se incluyeron además aspectos relativos a sus conocimientos y experiencia en videojuegos, además de su disponibilidad o facilidad para jugar determinado videojuego durante el taller.

Se realizó un primer acercamiento a sus hábitos lectores y a su experiencia previa en videojuegos por medio de preguntas sobre sus hábitos lectores y lecturas previas. También sobre el tiempo dedicado a los videojuegos, modos de juego y títulos preferidos; además sobre preferencias en lectura, géneros narrativos y actividades de ocio. Con ello fue posible, como ya se mencionó, conocer a los alumnos, sus conocimientos y experiencias previas en ambos temas y con ello se realizó la elección de material más adecuado, que fuera de su interés y lla mara su atención, pues como se mencionó, la lectura debe de ser entendida como un placer, un gusto voluntario.

Para la programación de actividades en el taller se tomó en cuenta la propuesta de la investigadora Gemma Lluch, presentada en el libro Del oral, audiovisual y digital a la lectura (y la escritura) en secundaria (2012) en donde se proponen actividades para el fomento y enseñanza de la literatura en alumnos de secundaria, por medio de narraciones audiovisuales tales como la televisión y el cine. Para este taller de literatura y videojuegos se retomaron algunas ideas como el análisis de ambas narraciones tomando en cuenta los siguientes puntos:

Identificar y valorar la caracterización de los personajes.

Identificar y valorar la estructura de la trama.

Identificar y valorar la ambientación.

Reconocer y valorar las elecciones de los elementos de un relato como el tipo de narrador, el punto de vista, la presentación de los personajes, etc. (Lluch 66).

Primeramente se plantearon algunas relaciones entre los videojuegos y los textos literarios para que percibieran la narración presente en los primeros. Los estudiantes realizaron Munguiay Araoz doi: https://doi.org/10.36799/el.v1i1.27 Volumen 1, Número 1, Año 2016, ISSN: $2448-5942$ 
algunas actividades en donde identificaron la narración en videojuegos, la estructura, personajes, tiempo, espacio y narrador, además de la presencia de temas.

Se buscó trabajar con videojuegos conocidos por los alumnos y textos literarios que compartieran elementos en común, tales como personajes, temas o género, ya que:

Los tópicos presentes en la historia de la literatura no son desconocidos por nuestros estudiantes, porque, aunque los formatos, las formas de elaboración y la complejidad no son las mismas, sí que están presentes en los relatos que consumen. Por lo tanto, puede ser una ayuda para introducirlos, para establecer puentes entre el mundo desconocido que plantea la literatura y el mundo próximo que crea el relato audiovisual a través de las diferentes pantallas (Lluch 75).

De esta manera, se introdujo al estudiante al mundo literario por medio del ocio, es decir por medio de una actividad absolutamente voluntaria y gustosa, como lo es jugar e interactuar con un videojuego.

La etapa inicial funcionó como introducción donde el estudiante, al interactuar con los videojuegos, hizo conciencia de las narraciones contenidas dentro de ellos, y de la relación que existe entre los videojuegos y otros medios como películas o programas de televisión e incluso relatos literarios clásicos y conocidos. Se manejó la narración como elemento primordial ya que es una de las principales formas de contacto que un posible lector tiene con el texto. Al respecto, Francisco Milla menciona que "una de las mejores maneras para animar a alguien a leer un libro o de ayudar a la elección libre de la lectura es contarle su argumento" (Milla 2001, 86). Así, mediante la narración desarrollada en el videojuego se intenta despertar el interés del alumno en otras formas de narrativa: la literatura.

Durante la primera semana se realizó una introducción para que los estudiantes comenzaran a reconocer los elementos principales dentro de una narración, tales como su estructura y los doce puntos principales presentes en una narración: equilibrio, llamada de aventura, rechazo de llamada, aparición de un mentor, travesía, pruebas, peligro, batalla, recompensa, retorno, resurrección, retorno exitoso (Lluch 16). ${ }^{9}$ Además de la estructura

\footnotetext{
${ }^{9}$ Ésta es una propuesta del autor Vladimir Propp, desarrollada en Morfología del cuento y retomada por Lluch para utilizarla con medios audiovisuales como lo es la televisión.

Munguiay Araoz doi: https://doi.org/10.36799/el.v1i1.27 Volumen 1, Número 1, Año 2016, ISSN: $2448-5942$
} 
también se tomaron en cuenta los elementos de tiempo-espacio, narrador y personajes. Los estudiantes reconocieron tales elementos en sus videojuegos favoritos, más adelante interactuaron con el videojuego Journey durante una sesión del taller. Después de jugarlo cada quién realizó su propia interpretación de la historia e identificó los elementos narrativos presentes en el juego.

La actividad con la cual concluyó la etapa primera fue con el videojuego Minecraft, cada uno de los estudiantes interactuó con el videojuego personalmente en su tiempo libre. En el taller escribieron la narración de su juego individual, jugando con los tipos de narrador, personajes y temas.

La segunda etapa del taller se desarrolló relacionando algunos textos literarios con videojuegos comerciales conocidos por los estudiantes. Esta etapa se comenzó a programar una vez que se conoció a los estudiantes, sus gustos y preferencias, de esta manera se les pudo ofrecer textos literarios que fueron de su agrado e interés. Posterior a la lectura se les brindó información de cada texto: fecha de publicación, datos del autor, textos similares, datos curiosos, etcétera. Esto para que el estudiante pudiera contextualizar y reflexionar sobre la vigencia de los textos en un mundo actual y, sobre todo, para hacerlos conscientes de que, a pesar de la distancia temporal que existe entre la publicación de los textos y los videojuegos elegidos, existe una fuerte relación entre ellos.

Durante la segunda etapa del taller se trabajó relacionando videojuegos y narración corta. Se inició con el género ciencia ficción, construyendo la relación entre cuentos de Isaac Asimov: Todos los males del mundo, Factor clave y de Harlan Ellison No tengo boca y debo gritar. Con videojuegos como Portal y Watch Dogs. Se estableció la relación de los personajes principales, tanto de las obras literarias como de los videojuegos: Multivac de Asimov, AM de Ellison, GLaDOS de Portal, y el sistema operativo ctOS presente en Watch Dogs. Los personajes máquina, vigilantes y con poder llevaron a seguir con la temática de distopías, trabajando con los mismos textos de Asimov y Ellison, además del cuento La pequeña guerra del mexicano Mauricio Schwarz y los videojuegos HalfLife, Bioshock, entre otros.

También se desarrolló el tema de viaje en el tiempo, los estudiantes leyeron cuentos de Ray Bradbury: El ruido de un trueno y El invento Kilimanjaro; y establecieron la relación con el 
videojuego The Legend of Zelda: Ocarina of Time. Compararon los viajes en el tiempo presentados en cada narración: motivos, medios y tiempo-espacio presentado.

Posteriormente se continuó trabajando en esta segunda etapa con narraciones y videojuegos de terror, los estudiantes leyeron La niebla de Stephen King haciendo una comparación con los videojuegos Silent Hill y Half Life, esta vez comparando personajes, tiempo-espacio y los elementos que causan miedo. Después los estudiantes leyeron las narraciones de H. P. Lovecraft: Herbert West: reanimador, y construyeron su relación con los videojuegos Resident Evil, Dead Islan, Dead Rising. También compararon el fenómeno zombi presentado en cada una de las narraciones, sus causas, el comportamiento y configuración de los personajes.

Por último, se programó una actividad para concluir el taller. Cada estudiante estableció la relación entre un texto literario y uno o varios videojuegos. Se les asignó una lectura individual y cada uno la leyó en su tiempo libre. Durante la última sesión del taller, describieron por escrito con qué videojuego encontraron relación y por qué, para después compartirlo con sus compañeros. El ejercicio obtuvo resultados positivos y fue más allá de las expectativas, pues los estudiantes realizaron la conexión entre el texto y los videojuegos durante la lectura de la narración, incluso algunos lograron relacionarlos con más de un videojuego.

Al terminar la actividad se realizó una pequeña reflexión sobre lo que se vio y se desarrolló en el taller. Se solicitó a cada estudiante una reflexión escrita sobre su experiencia en el taller; que describiera si encontraba algún cambio en cuanto a su actitud o en cuanto a su opinión sobre la literatura después de haber participado en él. Los resultados de la actividad fueron bastante alentadores, los estudiantes expresaron su opinión sobre la lectura de forma positiva, con un nuevo enfoque, anotando que durante las actividades del taller se percataron de la cercanía y familiaridad que hay entre la literatura y su mundo, los videojuegos en específico.

\section{RESULTADOS}

$\mathrm{Al}$ analizar las respuestas del cuestionario de reconocimiento que se aplicó al inicio del taller, los resultados llaman la atención en lo que respecta al apartado de lectura, y res ulta casi Munguiay Araoz doi: https://doi.org/10.36799/el.v1i1.27 Volumen 1, Número 1, Año 2016, ISSN: $2448-5942$ 
imposible no remitir a los datos registrados por las encuestas del Consejo Nacional para la Cultura y las Artes y de la Fundación Mexicana para el fomento de la lectura, pues la gran mayoría de los asistentes afirmaron estar interesados en la lectura: 10 de 12 asistentes respondieron afirmativamente, dos de forma negativa. Sin embargo, cuando se ahonda un poco más en la cuestión de hábitos lectores resalta que la lectura realizada no se trata de textos literarios, las más recurrentes fueron: artículos en internet, cómics y textos científicos.

$\mathrm{Al}$ momento de preguntar sobre el tiempo dedicado a la lectura, la respuesta promedio fue de una hora al día. Entre las razones anotadas por las cuales se le dedica nada o poco tiempo a la lectura fueron en su mayoría por la falta de tiempo.

Sin embargo, más adelante, al momento de preguntar sobre su experiencia en videojuegos y preguntar por las horas diarias dedicadas a la interacción de videojuegos, las respuestas oscilaron entre de 2 a 3 horas y más de 4 horas.

Al reflexionar sobre los resultados de estos cuestionarios queda clara la existencia de ese nuevo tipo de lectores, quienes afirman leer y tener interés en la lectura, pero evidencian que leen poco y que lo leído no son textos literarios.

Por ello, el taller de literatura y videojuegos buscó llamar la atención del estudiante y llevarlo hacia la lectura literaria. Se puede a firmar que durante las actividades del taller la actitud de los jóvenes frente a textos literarios fue positiva. Incluso se registra ron casos en donde la opinión hacia la lectura cambió de ser negativa a totalmente positiva.

Consideramos que el taller de literatura y videojuegos, a pesar de su corta duración, logró su objetivo, pues los estudiantes mostraron mucho entusiasmo ante los textos literarios presentados durante el curso del taller. Al finalizar la última sesión, todos se mostraron interesados en la continuación del taller durante el semestre siguiente. Algunos estudiantes han dado muestras de seguir su interés en lectura, y a través de las redes sociales publican adquisiciones de libros, comparten videos en donde se habla sobre la configuración de personajes y la narración en videojuegos.

Sus acciones representan de cierta manera el éxito del taller, ya que se logró el objetivo de acercar a los estudiantes al mundo de la lectura literaria, se demostró que la litera tura no es 
ajena al mundo en el que se desarrollan, logrando así un cambio de actitud de los estudiantes hacia la literatura.

Como acciones a futuro se pretende desarrollar el mismo taller por un tiempo más prolongado y así poder utilizar obras literarias más extensas, como novelas. De esta manera será posible crear un vínculo más fuerte entre el estudiante y la lectura. De igual forma, sería interesante aplicar el mismo método pero con grupos diferentes, como niños, adolescentes y jóvenes adultos fuera de una institución universitaria, pues creemos que se tienen todos los elementos para lograr resultados positivos.

Finalmente, consideramos necesaria desarrollar y utilizar nuevas estrategias para atraer a nuevos lectores. Los videojuegos pueden funcionar como una excelente herramienta para lograr tales objetivos ya que son narraciones interactivas y contienen los elementos bá sicos de un texto literario. Asimismo, llaman la atención y tienen una gran aceptación entre los niños, jóvenes y adultos, y por supuesto, son de fácil acceso.

Los estudiantes han tenido contacto con los videojuegos desde hace mucho tiempo, por sus edades y la era en que les tocó vivir, muchos de ellos han crecido conviviendo con ellos día a día. Utilicemos entonces los videojuegos como herramienta para fomentar el gusto por la literatura. No olvidemos que, como dice García Galeano, "La literatura, el arte en general, puede operar en la sociedad para transformarla” $(2001,339)$.

\section{REFERENCIAS BIBLIOGRÁFICAS}

Castronovo, Adela. Nuevas propuestas en promoción de la lectura. Argentina: Ediciones Colihue, 2007.

Cassany, Daniel, Martha Luna y Gloria Sanz. Enseñar lengua. México: Graó, 2007.

García Galeano, Ángel. "Didáctica de la literatura ” Nueva Introducción a la teoría de la Literatura. Miguel Ángel garrido. Madrid: Síntesis. (2001):317-347.

Gobierno de los Estados Unidos Mexicanos. Encuesta Nacional de Lectura. México: Consejo Nacional para la Cultura y las Artes. 2006.

Munguiay Araoz doi: https://doi.org/10.36799/el.v1i1.27 Volumen 1, Número 1, Año 2016, ISSN: $2448-5942$ 
Cerrillo, Pedro. "Los nuevos lectores: formación del lector literario" Universidad de Castilla La Mancha. Alicante: Biblioteca Virtual de Cervantes, 2007.

Fundación Mexicana para el fomento de la lectura. De la penumbra a la oscuridad. Encuesta Nacional de Lectura. México: FunLectura. 2012. http://www.lector.mx/images/noticias/1.\% 20ENL\% 202012\%20LR.pdf

Lluch, Gemma. Del oral, audiovisualy digital a la lectura (y escritura) en secundaria. Madrid: Fundación SM, 2012.

Martín Vegas, Rosa Ana. Manual de didáctica de la lengua y la literatura. España: Síntesis. 2010.

Milla, Francisco. Actividades Creativas Para La Lecto-Escritura. México: Alfaomega. 2001.

Villamil, Jenaro. "Entre 108 países, México es penúltimo lugar en lectura”. Proceso. 2013. Consultada el 14 abril 2013. http://www.proceso.com.mx/?p=339874

Munguiay Araoz doi: https://doi.org/10.36799/el.v1i1.27 Volumen 1, Número 1, Año 2016, ISSN: $2448-5942$ 